\title{
Optimal Time Interval between Laparoscopic Tubal Ligation for Hydrosalpinges and ICSI-ET
}

\author{
Shubhadeep Bhattacharjee
}

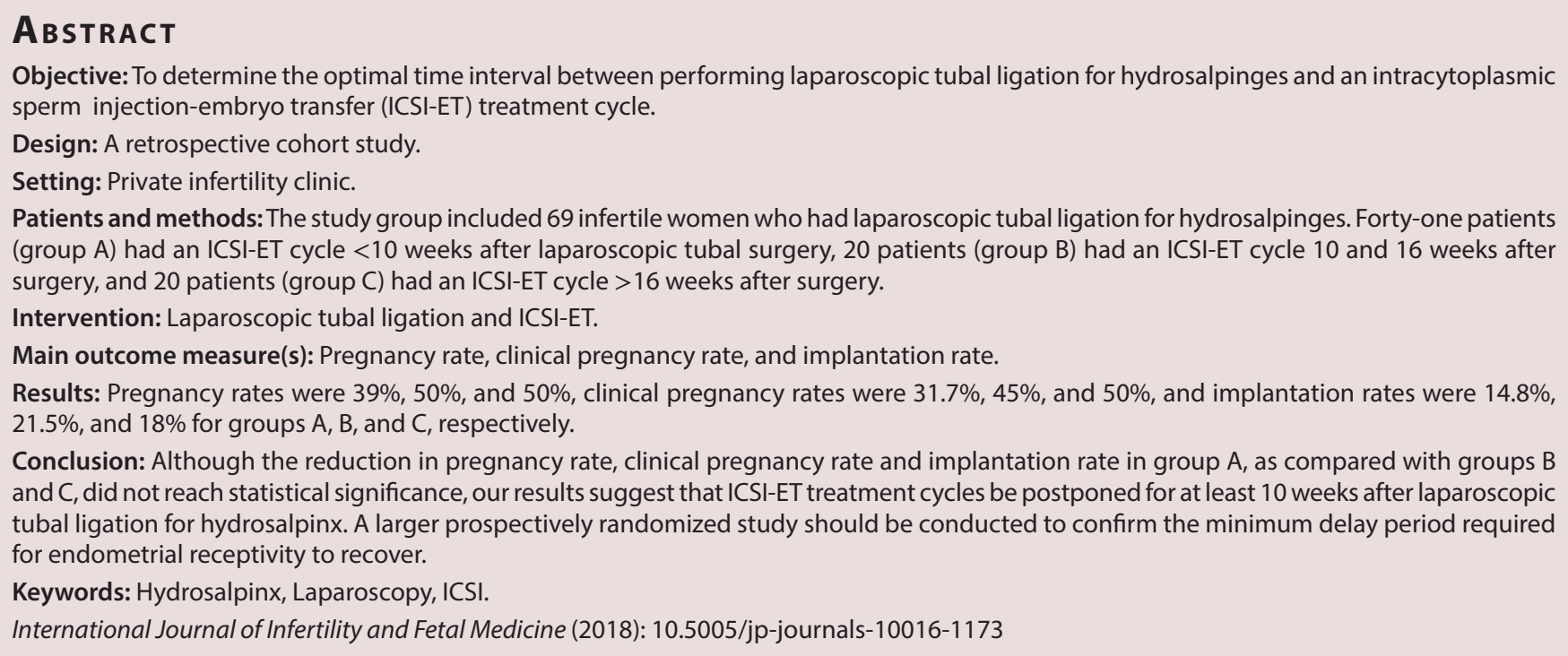

\section{INTRODUCTION}

Tubal disease is one of the major indications for in vitro fertilization (IVF). The etiology of the tubal factor infertility may, however, have a significant impact on patient fecundity. Hydrosalpinx associated tubal factor has been shown to be predisposed to the most impaired reproductive outcomes of the tubal factors. This has been inconclusively demonstrated by a number of studies that have shown a significant reduction in pregnancy rates and increase in pregnancy loss in patients with hydrosalpinges, ${ }^{1-8}$ especially, in cases where the hydrosalpinges are large enough to be visible on ultrasound. ${ }^{8}$ The deleterious impact of hydrosalpinx on reproduction maybe as a direct result of the tubo-utero circulation of hydrosalpinx fluid, as the fluid has been linked to embryo cytotoxicity, altered embryo-endometrium receptivity, and altered tubo-uterine flow dynamics. ${ }^{9-14}$

With advances in assisted reproductive technologies (ARTs), it has become usual practice to attempt to treat these patients with IVF rather than to attempt to restore tubal function for natural conception. However, to accomplish this, patients have to undergo permanent tubal sterilization procedures that block the tubo-utero connection and therefore, the flow of hydrosalpinx fluid into the uterus. Numerous studies have inconclusively shown that surgical interventions such as tubal ligation or proximal tubal occlusion significantly improve assisted reproduction outcomes in IVF. ${ }^{15-18}$

No studies have, however, investigated whether the hydrosalpinx fluid has a lasting effect on the clinical aspects endometrial receptivity. The purpose of this retrospective cohort study was therefore to indirectly investigate the length of time
Department of Reproductive Medicine, Indira IVF Hospital, New Delhi, India

Corresponding Author: Shubhadeep Bhattacharjee, Department of Reproductive Medicine, Indira IVF Hospital, New Delhi, India, e-mail: shubhadeep96bhattacharjee@gmail.com

How to cite this article: Bhattacharjee S. Optimal Time Interval between Laparoscopic Tubal Ligation for Hydrosalpinges and ICSI-ET. Int J Infertil Fetal Med 2018;9(3):37-40.

Source of support: Nil

Conflict of interest: None

needed for the uterine endometrium to recovery from the effects of hydrosalpinx by analyzing the pregnancy outcomes at different time periods post-tubal surgery.

\section{Patients and Methods}

Patients who were diagnosed with hydrosalpinx-related tubal factor infertility with the use of transvaginal ultrasound or hysterosalpingography at Indira IVF, New Delhi and who wished to pursue IVF, during March 2016 to May 2017 were consulted on the need for and the implications of laparoscopic tubal ligation. All patients received counseling on the implications and risks of the procedures and informed consents were obtained before the therapeutic tubal procedures were scheduled to be performed. Patients excluded from further analysis were patients older than 42 , patients with only one ovary, and patients with a basal FSH concentration of $>12 \mathrm{IU} / \mathrm{l}$. Eighty-one infertile patients who met 
the criteria underwent laparoscopic tubal ligation procedures for hydro-salpinges during the study period. Institutional ethics committee approval was received for the study. Laparoscopic uni- or bilateral tubal ligation procedures were performed as ambulatory procedures at Indira IVF. Laparoscopic tubal ligation was performed by bipolar cautery of affected tubes at the proximal position followed by the cutting of the tube at the coagulated area, leaving the cut tube in place. Two hours postoperatively all patients were assessed before being discharged.

All patients at Indira IVF undergo ICSI-ET cycles. Controlled ovarian stimulation (COS) was performed using a $\mathrm{GnRH}$ agonist protocol, with the administering of luteal $\mathrm{GnRH}$ agonist (Leuprolide acetate; Lucrin daily, Abbott, Turkey) and recombinant gonadotropins (Gonal F; Serono, or Puregon; Organon). An hCG (Ovidrel, Merck Serona, $2501 \mathrm{~g} / 0.05 \mathrm{~mL}$ ) trigger was administered when at least three follicles reached $17 \mathrm{~mm}$ in diameter. Transvaginal ultrasound-guided oocyte retrieval was performed 36 hours after ovulation induction. Retrieved oocytes were denuded and all mature oocytes were fertilized by ICSI. All embryo replacements were performed on day 2 of embryo development. Embryos were graded on a scale of 1-4 (1 being best) based on cell number, blastomere size and equivalence, and percentage of fragmentation. ${ }^{19}$ The luteal phases of all cycles were supplemented with E2 (Estrofem, Novo Nordisk, 2 mg BD) and P4 (Crınone, Merck Serono, $8 \% \mathrm{BD}$ ) and continued for at least 9 weeks of gestation if pregnant. The patients were divided into three groups according to interval in weeks between laparoscopic tubal ligation and ICSI-ET treatment; group A included all patients who had ICSI-ET cycles $<10$ weeks after laparoscopic surgery, group B included all patients who had ICSI-ET cycles 10 to16 weeks after surgery, and group C included all patients who had ICSI-ET cycles $>16$ weeks after surgery.

\section{Statistical Analysis}

Age, percentages of patients over 37 years of age, bilaterality, antral follicle count, length of infertility, day $3 \mathrm{FSH}$, peak E2 levels, days of stimulation, total rFSH dosage, total oocytes retrieved, $\mathrm{M} 2$ oocytes retrieved, number of embryos transferred, number of grade 1-embryos transferred, pregnancy rate (positive HCG), clinical pregnancy rate (positive fetal cardiac activity), and implantation rate were compared between the groups.

ANOVA and Chi-square test were used for statistical comparisons. $p<0.05$ was considered statistically significant. Statistical calculations were performed using Sigmastat for Windows, version 3.0 (Jardel Scientific Corporation, San Ra-fael, CA).

\section{Results}

Forty-one patients (group A) had ICSI-ET cycles $<10$ weeks after laparoscopic surgery, 20 patients (group B) had ICSI-ET cycles 10 to 16 weeks after surgery and 20 patients (group C) had ICSI-ET cycles $>16$ weeks after surgery. The demographical characteristics and reproduction outcomes of the three patient groups are presented and described in Table 1. No statistically significant differences were found between the demographic and reproductive variables of age, bilaterality, antral follicle count, day 3 FSH, peak E2 levels, days of stimulation, total rFSH dosage age, total oocytes retrieved, MIl oocytes retrieved, number of embryos transferred, number of grade 1-embryos transferred between the three groups.

The pregnancy outcomes for groups $A$ to $C$ were as follows; positive b hCG pregnancy ( 39.0 vs 50 vs $50 ; p=0.610$ ), clinical pregnancy rate ( 31.7 vs 45.0 vs $50.0 ; p=0.330$ ), and implantation rate (14.8 vs 21.5 vs $18.0 ; p=0.420$ ). Although not significant, all the parameters in group $A$ were reduced compared to groups $B$ and $C$, while outcomes between groups $B$ and $C$ were very similar.

\section{Discussion}

Tubal disease is one of the major causes of female infertility. ${ }^{20}$ IVF-embryo transfer was initially developed as a method to overcome tubal infertility. ${ }^{21}$ IVF-embryo transfer studies examining reproductive outcomes in tubal disease patients reported particularly low pregnancy and implantation rates in patients with hydrosalpinx associated tubal disease. ${ }^{5,7,8}$ Therapeutic studies have shown that with appropriate surgical intervention in vivo fecundity in affected patients can be improved, ${ }^{3,17}$ as well as in vitro fecundity, conclusively demonstrated in a prospective, randomized

Table 1: Statistical analyses of variables

\begin{tabular}{llllc}
\hline & Group $A<10$ weeks & Group B 10-16 weeks & Group C >16 weeks & $p$-value \\
\hline No. of patients & 41 & 20 & 20 & NA \\
Age (in years) & $32.8 \pm 0.6$ & $33.6 \pm 0.8$ & $32.9 \pm 0.8$ & 0.709 \\
Length of infertility (years) & $7.5 \pm 0.6$ & $6.5 \pm 0.6$ & $7.1 \pm 0.8$ & 0.583 \\
Bilateral tubal occlusion (\%) & 36.5 & 40.0 & 40.0 & 0.951 \\
No. of antral follicles $(n)$ & $13.2 \pm 0.9$ & $13.9 \pm 1.2$ & $13.3 \pm 1.1$ & 0.892 \\
Day 3 FSH (IU/mL) & $6.9 \pm 0.6$ & $7.2 \pm 0.8$ & $6.9 \pm 0.7$ & 0.950 \\
Peak E2 levels $(\mathrm{pg} / \mathrm{mL})$ & $1605 \pm 405$ & $1780 \pm 350$ & $1780 \pm 350$ & 0.963 \\
Days of stimulation $(n)$ & $8.8 \pm 0.2$ & $9.5 \pm 0.3$ & $9.0 \pm 0.4$ & 0.258 \\
FSH ampoules $(n)$ & $53.1 \pm 3.4$ & $48.1 \pm 5.9$ & $58.1 \pm 4.1$ & 0.356 \\
Oocytes collected $(n)$ & $13.7 \pm 0.9$ & $14.6 \pm 1.7$ & $121.8 \pm 1.1$ & 0.665 \\
Mll oocytes collected $(n)$ & $9.8 \pm 0.8$ & $9.8 \pm 1.4$ & $9.3 \pm 1.2$ & 0.952 \\
Embryos transferred $(n)$ & $2.3 \pm 0.1$ & $2.0 \pm 0.3$ & $2.5 \pm 0.4$ & 0.414 \\
Grade 1 embryos $(n)$ & $2.0 \pm 0.2$ & $1.8 \pm 0.3$ & $2.2 \pm 0.3$ & 0.629 \\
Pregnancy rates $(\%)$ & 39.0 & 50.0 & 50.0 & 0.610 \\
Clinical pregnancy rates $(\%)$ & 31.7 & 45.0 & 50.0 & 0.330 \\
Implantation rates $(\%)$ & 14.8 & 21.5 & 18.0 & 0.420 \\
\hline
\end{tabular}

Note: values are mean \pm SEM 
multi-center trial to measure the benefit of tubal surgical intervention prior to IVF on clinical reproductive outcomes. ${ }^{15}$

Direct embryotoxic effects, ${ }^{9}$ physical interference due to the intrauterine accumulation of refluxed fluid, ${ }^{14}$ and an altered endometrial receptivity ${ }^{13}$ are among some of the proposed pathological mechanisms by which hydrosalpinx impairs intrauterine embryo development, embryo implantation and pregnancy development. Lessey et al. ${ }^{22,23}$ and Tabibzadeh ${ }^{24}$ evaluated the expression of integrin molecules by human endometrium throughout the menstrual cycle. The vitronectin receptor, avb3, was found to appear abruptly on cycle day 19 or 20, coincident with the opening of the putative implantation window.

The expression of avb3 was absent in endometrial biopsies taken on these days when there was maturational delay. Avb3 integrin may therefore serve as an important internal marker for luteal phase maturation and endometrium receptivity. ${ }^{22,23}$ Meyer et al. ${ }^{13}$ documented endometrial dysfunction in patients with hydrosalpinges, with the histological samples of affected patients exhibiting the signs of maturational delay and lower integrin expression levels. Furthermore, Lessey et al. ${ }^{23}$ demonstrated in their study that tubal corrective surgery in patients with confirmed tubal hydrosalpinges and concomitant reduced avb3 expression had avb3 expression levels return to normal 3 months postoperatively. Similarly, Bildirici et al. ${ }^{25}$ demonstrated a significant increase in avb3 integrin expression following salpingectomy and therefore argued that this increased expression would be an indicative of improved endometrial receptivity.

Our study was therefore initiated to test the hypothesis that a period of at least 3 months postoperatively was required for endometrial receptivity to recover from the effects of hydrosalpinx. The key objective being to determine the minimum length of time required to allow the complete wash-out of the hydrosalpinx related effects on clinical pregnancy outcomes following tubal surgery. In our study period, 81 patients were found who had tubal ligations for uni- or bilateral hydrosalpinx, because of their wish to have assisted reproductive treatment. The ICSI-ET treatment cycles performed for these patients were categorized according to three delay intervals ( $<10$ weeks, $10-16$ weeks, and $>16$ weeks) between surgery and treatment and the inter-category reproductive outcomes compared. The results of the study showed that a minimum 10 week wash-put period should be allowed, as patients treated within this period had reduced reproductive outcomes as compared to the patients treated after 10 weeks. The results of our clinical study corroborates the evidence found in the integrin expression studies that there is a time-dependent delay of at least 10 weeks for the endometrial development and function to normalize after the removal of the effects of hydrosalpinx The results from our retrospective study are unfortunately only strongly suggestive of improved reproductive outcomes, if ICSI-ET treatment was performed at least 10 weeks after laparoscopic surgery for hydrosalpinx, as the differences in reproductive outcomes did not reach statistical significance.

The outcomes of this study and those of the integrin expression studies do, however, suggest that a larger prospectively randomized study is warranted to confirm the minimum delay period required for the endometrium to recover from the effects of hydrosalpinx.

\section{References}

1. Nackley AC, Muasher SJ. The significance of hydrosalpinx in in vitro fertilization. Fertil Steril 1998;69:373-384. DOI: 10.1016/S00150282(97)00484-6.

2. Camus $E$, Poncelet $C$, et al. Pregnancy rates after in vitro fertilization in cases of tubal infertility with and without hydrosalpinx: a metaanalysis of published comparative studies. Hum Reprod 1999;14:12431249. DOI: 10.1093/humrep/14.5.1243.

3. Vandromme J, Chasse E, et al. Hydrosalpinges in in-vitro fertilization: an unfavorable prognostic feature. Hum Reprod 1995;10:579-589. DOI: 10.1093/oxfordjournals.humrep.a135992.

4. Wainer $\mathrm{R}$, Camus $\mathrm{E}$, et al. Does hydrosalpinx reduce the pregnancy rate after in vitro fertilization? Fertil Steril 1997;68:1022-1026. DOI: 10.1016/S0015-0282(97)00405-6.

5. Katz E, Akman M, et al. Deleterious effect of the presence of hydrosalpinx on implantation and pregnancy rates with in vitro fertilization. Fertil Steril 1996;66:122-125. DOI: 10.1016/S00150282(16)58398-8.

6. Akman MA, Garcia JE, et al. Hydrosalpinx affects the implantation of previously cryopre-served embryos. Hum Reprod 1996;11:1013-1014. DOI: 10.1093/oxfordjournals.humrep.a019287.

7. Strandell $A$, Waldenstrom $U$, et al. Hydro-salpinx reduces in-vitro fertilization/embryo transfer pregnancy rates. Hum Reprod 1994;9:861-863. DOI: 10.1093/oxfordjournals.humrep.a138606.

8. Andersen AN, Yue Z, et al. Low implantation rate after in vitro fertilization in patients with hydrosalpinges diagnosed by ultrasonography. Hum Reprod 1994;9:1935-1938. DOI: 10.1093/ oxfordjournals.humrep.a138362.

9. Mukherjee $T$, Copperman AB, et al. Hydrosalpinx fluid has embryotoxic effects on murine embryogenesis: a case for prophylactic salpingectomy. Fertil Steril 1996;66:851-853. DOI: 10.1016/S0015-0282(16)58652-X.

10. Beyler $\mathrm{SA}$, James KP, et al. Hydrosalpingeal fluid inhibits in-vitro embryonic development in a murine model. Hum Reprod 1997;12:2724-2728. DOI: 10.1093/humrep/12.12.2724.

11. Schenk LM, Ramey JW, et al. Embryo toxicity of hydrosalpinx fluid. Presented at the 43rd annual meeting of the Society of Gynecologic Investigation 1996 (abstract). J Soc Gynecol Invest 1996;3:90A.

12. Rawe VJ, Liu J, et al. Effect of human hydrosalpinx fluid on murine embryo development and implantation. Fertil Steril 1997;68:668-670. DOI: 10.1016/S0015-0282(97)00319-1.

13. Meyer WR, Castelbaum AJ, et al. Hydrosalpinges adversely affect markers of endometrial receptivity. Hum Reprod 1997;12:1393-1398. DOI: 10.1093/humrep/12.7.1393.

14. Mansour RT, Aboulghar MA, et al. Fluid accumulation of the uterine cavity before embryo transfer: a possible hindrance for implantation. $J$ In Vitro Fert Embryo Trans 1991;8:157-159. DOI: 10.1007/BF01131707.

15. Strandell A, Lindhard A, et al. Hydrosalpinx and IVF outcome: a prospective randomized multicentre trial in Scandinavia on salpingectomy prior to IVF. Hum Reprod 1999;14:2762-2769. DOI: 10.1093/humrep/14.11.2762.

16. Bredkjaer $\mathrm{HE}$, Ziebe $\mathrm{S}$, et al. Delivery rates after in-vitro fertilization following bilateral salpingectomy due to hydrosalpinges: a case control study. Hum Reprod 1999;14:101-105. DOI: 10.1093/ humrep/14.1.101.

17. Murray DL, Sagoskin AW, et al. The adverse effect of hydrosalpinges on in vitro fertilization pregnancy rate and the benefit of surgical correction. Fertil Steril 1998;69:41-45. DOI: 10.1016/S00150282(97)00447-0.

18. Surrey ES, Schoolcraft WB. Laparoscopic management of hydrosalpinges before in vitro fertilization-embryo transfer: salpingectomy vs proximal tubal occlusion. Fertil Steril 2001;75: 612-617. DOI: 10.1016/S0015-0282(00)01742-8. 
19. Berkkanoglu $M$, Isikoglu $M$, et al. Flushing the endometrium prior to the embryo transfer does not affect the pregnancy rate. RBM Online 2006;13:268-271. DOI: 10.1016/S1472-6483(10)60625-6.

20. Westrom L.Incidence, prevalence, and trends ofacute pelvicinflammatory disease and its consequences in industrialized countries. Am J Obstet Gynecol 1980;138:880-892. DOI: 10.1016/0002-9378(80)91077-7.

21. Edwards RG, Fishel SB, et al. Factors influencing the success of in vitro fertilization alleviating human infertility. J In Vitro Fertil Embryo Trans 1984;1:3-23. DOI: 10.1007/BF01129615.

22. Lessey BA, Damjanovich $L$, et al. Integrin adhesionmolecules in the human endometrium. J Clin Invest 1992;90:188-195. DOI: 10.1172/ JCI115835.
23. Lessey BA, Castelbaum AJ, et al. Further characterization of endometrial integrins during the menstrual cycle and in pregnancy. Fertil Steril 1994;62:497-506. DOI: 10.1016/S0015-0282(16) 56937-4.

24. Tabibzadeh S. Patterns of expression of integrin molecules in human endometrium throughout the menstrual cycle. Hum Reprod 1992;7:876-882. DOI: 10.1093/oxfordjournals.humrep. a137753.

25. Bildirici I, Bukulmez O, et al. A prospective evaluation of the effect of salpingectomy on endometrial receptivity in cases of women with communicating hydrosalpinges. Hum Reprod 2001;16:2422-2426. DOI: 10.1093/humrep/16.11.2422. 